\title{
Pancreatic-type acinar cell carcinoma of the liver: a clinicopathologic study of four patients
}

\author{
Abbas Agaimy ${ }^{1}$, Annette Kaiser ${ }^{2}$, Karen Becker ${ }^{3}$, Jan-Hinrich Bräsen ${ }^{4}$, Peter H Wünsch ${ }^{2}$, \\ N Volkan Adsay ${ }^{5}$ and Günter Klöppel ${ }^{3,4}$ \\ ${ }^{1}$ Institutes of Pathology, University of Erlangen, Erlangen, Germany; ${ }^{2}$ Klinikum Nürnberg, Nürnberg, \\ Germany; ${ }^{3}$ Technical University of München, München, Germany; ${ }^{4}$ University of Kiel, Kiel, Germany and \\ ${ }^{5}$ Department of Surgical Pathology, Emory University Hospital, Atlanta, GA, USA
}

\begin{abstract}
Acinar cell carcinoma of pancreatic type rarely occurs at extra-pancreatic sites. We report four primary liver tumors with features of pancreatic acinar cell carcinoma. The patients were two males and two females with a mean age of 65 years (range, 49-72 years). They had upper abdominal pain, weight loss and/or an incidentally discovered liver mass. None had evidence of a primary pancreatic tumor. Grossly, the tumors were large (mean size, $12 \mathrm{~cm}$ ), well circumscribed and showed a lobulated cut surface. Histologically, they showed a predominantly microacinar pattern, with occasional trabecular, solid and microcystic areas. Cellular atypia and mitotic activity varied within the same tumor and from tumor to tumor. Immunohistochemically, the tumor cells were positive for cytokeratin 18 and at least one acinar cell marker (ie, trypsin, amylase or lipase), but were negative for cytokeratins 7, 19 and 20, HepPar-1, AFP, CD10, carcinoembryonic antigen, CD56, Islet-1 and CDX2. Two tumors stained focally for synaptophysin and chromogranin A. Adjacent liver parenchyma displayed no evidence of cirrhosis. During a mean follow-up of 22 months (range, 3-38 months) no metastases occurred, but one patient developed local recurrence. Our study demonstrates that acinar cell carcinoma of pancreatic type may also originate from the liver and can be readily distinguished from other primary liver neoplasms by its distinct histological and immunohistochemical features. Because our cases were observed within a rather short period, it is likely that this tumor type is so far underrecognized and has been mistaken as a variant of hepatocellular carcinoma, cholangiocarcinoma or any other liver tumor.

Modern Pathology (2011) 24, 1620-1626; doi:10.1038/modpathol.2011.127; published online 12 August 2011
\end{abstract}

Keywords: acinar cell carcinoma; differential diagnosis; immunohistochemistry; liver neoplasms; pancreas

Acinar cell carcinoma of the pancreas is a rare aggressive neoplasm constituting $1-2 \%$ of pancreatic cancers. ${ }^{1}$ These neoplasms affect mostly adults with a mean age of 58.3 years but with a wide age range (10-87 years). ${ }^{1}$ They show a striking predilection for men (male to female ratio 3.6:1). ${ }^{1}$ Approximately half of the patients have evidence of metastatic disease at the time of diagnosis, underlining their aggressive behavior. ${ }^{2}$ Besides their characteristic histological features, acinar cell carcinomas are defined on the basis of their immunohistochemical staining for pancreatic enzymes,

Correspondence: Dr A Agaimy, MD, Pathologisches Institut, Universitätsklinikum Erlangen, Krankenhausstraße 12, 91054 Erlangen, Germany.

E-mail: abbas.agaimy@uk-erlangen.de

Received 13 April 2011; revised 14 June 2011; accepted 15 June 2011; published online 12 August 2011 notably trypsin. ${ }^{3-5}$ There are few case reports on neoplasms with pancreatic acinar cell differentiation that occurred outside the pancreas, particularly in the gastrointestinal tract. ${ }^{6-8}$ Some of these cases were found in or next to heterotopic ${ }^{9,10}$ or metaplastic $^{11}$ pancreatic tissue that was considered a possible source of the tumor. The occurrence of an acinar cell carcinoma as a primary liver neoplasm has been, so far, only once reported. ${ }^{12}$ In this study, we describe four primary liver carcinomas that show acinar cell differentiation without association with any other well established liver tumor component, thus justifying their classification as primary acinar cell carcinoma of the liver. A primary pancreatic tumor was excluded on preoperative imaging procedures, during surgery and on through diagnostic workup after surgery using computed tomography (CT) scan, ultrasound and magnetic resonance imaging in all cases. 


\section{Materials and methods}

The four tumors of this series were observed during the last 4 years. All cases were initially diagnosed as 'neoplasm with pancreatic acinar differentiation' and none of the cases was retrieved from archival tissue or by retrospective reevaluation of liver tumor series. Accordingly, it is not possible to make any suggestions regarding the frequency of this tumor type in our material. In all four patients the tumors were completely resected. The resected specimens were extensively sectioned with a minimum of 10 blocks per tumor. Tissue samples were fixed overnight in $4 \%$ buffered formalin, processed routinely and stained with hematoxylin \& eosin, Periodic acid-Schiff (PAS), Prussian blue, reticulin and trichrome stains. Immunohistochemistry was performed using the following antibodies: pankeratin (KL-1, 1:200, Beckman-coulter GmbH, Krefeld, Germany), EMA (clone E29, 1:200, DAKO, Hamburg, Germany), CK5/6 (clone D5/16B4, 1:50, Zytomed, Berlin, Germany), CK7 (clone OV-TL 12/30, 1:1000, Biogenex), CK18 (clone CY-90, 1:500, Sigma), CK19 (clone RCK108, 1:300, DAKO), CK20 (clone Ks 20.8, 1:50, DAKO), TTF-1 (clone 8G7G3/1, 1:500, Zytomed), HepPar-1 (clone OCH1E5, 1:200, DAKO), AFP (polyclonal, 1:20, DAKO), CD10 (clone 56C6, 1:50, Novocastra), carcinoembryonic antigen (CEA, polyclonal, 1:500, DAKO), CD56 (clone 1B6, 1:50, Novocastra), synaptophysin (clone SY38, 1:50, DAKO), chromogranin A (clone LK2H10, 1:500, Beckman-coulter GmbH), CDX2 (clone CDX2-88, 1:200, Biogenex), Islet-1 (clone 1H9, 1:1000, Abcam), amylase (clone A8273, 1:1000, Sigma), lipase (clone 4I2, 1:2000, Zytomed), trypsin (1:100, Ventrex Laboratories, Portland, OR, USA) and Ki-67 (MiB-1, 1:100, DAKO).

\section{Results}

\section{Case Histories}

\section{Case 1}

A 68-year old woman with a history of diabetes mellitus type 2 and chronic alcohol abuse presented with unexplained weight loss. Liver function test and tumor markers (AFP, CEA) were normal. Abdominal ultrasound and CT scan revealed a $7-\mathrm{cm}$ well circumscribed mass in the left liver lobe (segment III) that was suspicious for hepatocellular carcinoma. Rectocolonoscopy showed two small adenomas in the descending colon, which were removed by endoscopic polypectomy. The liver tumor was completely resected. Intra-operatively, there was neither evidence for local or peritoneal tumor spread, nor was a pancreatic mass detected. Fourteen months later, the patient was diagnosed with a laryngeal squamous cell carcinoma and received appropriate surgery. At last follow-up, 38 months after liver surgery, the patient had no evidence of metastasis or pancreatic tumor.

\section{Case 2}

A 71-year old man with abdominal pain was diagnosed with a liver mass in the right lobe. Staging procedures including whole-body CT scan, endoscopy, ENT-panendoscopy, bronchoscopy and octreotide scintigraphy revealed no other primary tumor. His previous medical history disclosed arterial hypertension and nicotine abuse, but no other significant or relevant findings. Clinical diagnosis was 'suspicious for hepatocellular carcinoma'. A right-sided hemihepatectomy with hepatico jejunostomy (Y-Roux) was performed. Intra-operatively there was no evidence of any primary pancreatic tumor. Postoperatively the patient developed pathological liver function tests with evidence of cholestasis and ascites. To exclude diffuse micrometastasis, a liver biopsy was obtained and displayed significant cholestasis but no tumor tissue. The patient died 3 months later without evidence of metastatic tumor spread. An autopsy was not performed.

\section{Case 3}

A 72-year old man presented with non-specific abdominal discomfort. The patient had no liverrelevant clinical history. Imaging studies demonstrated a large right-sided liver mass that was clinically suspicious for cholangiocarcinoma. The remainder of the liver demonstrated no evidence for chronic liver disease or additional mass lesions. The tumor was completely resected. Intra-operatively, there was no evidence of any primary pancreatic tumor. Eighteen months later, the tumor recurred in the left liver lobe and was treated by chemotherapy. Twenty months after the initial diagnosis the patient is alive with localized recurrent disease.

\section{Case 4}

A 49-year old woman with increasing abdominal discomfort and pain was found to have a hepatic mass occupying large portions of segment $\mathrm{V}$ and segment VIII. On contrast-enhanced CT scan, the tumor showed markedly irregular outlines, profound peripheral enhancement and central necrosis. Several small nodular foci were identified peripherally. The tumor displaced the adjacent vasculature but showed no frank invasion per imaging analysis. There was minimal adjacent biliary ductal dilatation within the right hepatic lobe. The remainder of the liver demonstrated no evidence for chronic liver disease or additional mass lesion. The pancreas, adrenals, spleen and abdominal hollow viscous organs were unremarkable. No abdominal lymphadenopathy or major vascular occlusion was identified. The patient underwent a cholecystectomy and resection of the right liver lobe. There was no evidence of any primary pancreatic tumor, neither intra-operatively nor with subsequent imaging analysis. The patient is currently alive and well with no evidence of disease 28 months after initial diagnosis. 


\section{Pathological Findings}

\section{Gross features}

The tumor sizes ranged from $7 \mathrm{~cm}$ to $19 \mathrm{~cm}$ in maximum diameter (mean size, $12 \mathrm{~cm}$ ). They displayed well-circumscribed nodular gray-whitish firm cut-surfaces with remarkable gross lobulation (Figure 1a). Some tumors also contained areas of cystic changes, occasionally associated with focal hemorrhage and necrosis (Figure 1b). The surrounding liver parenchyma was grossly unremarkable. The resection margins were free of tumor tissue in all cases.

\section{Histological findings}

The four tumors showed essentially similar features. At low magnification, the tumors formed large nodular lobules separated by broad vascularised fibrous septa occasionally containing a few bile ductules at the septal-nodular interface. The tumor lobules were generally well circumscribed peripherally (Figure 2a). The tumor cells were predominantly arranged in small well-organized acini with small lumens reminiscent of pancreatic acinar tissue at low
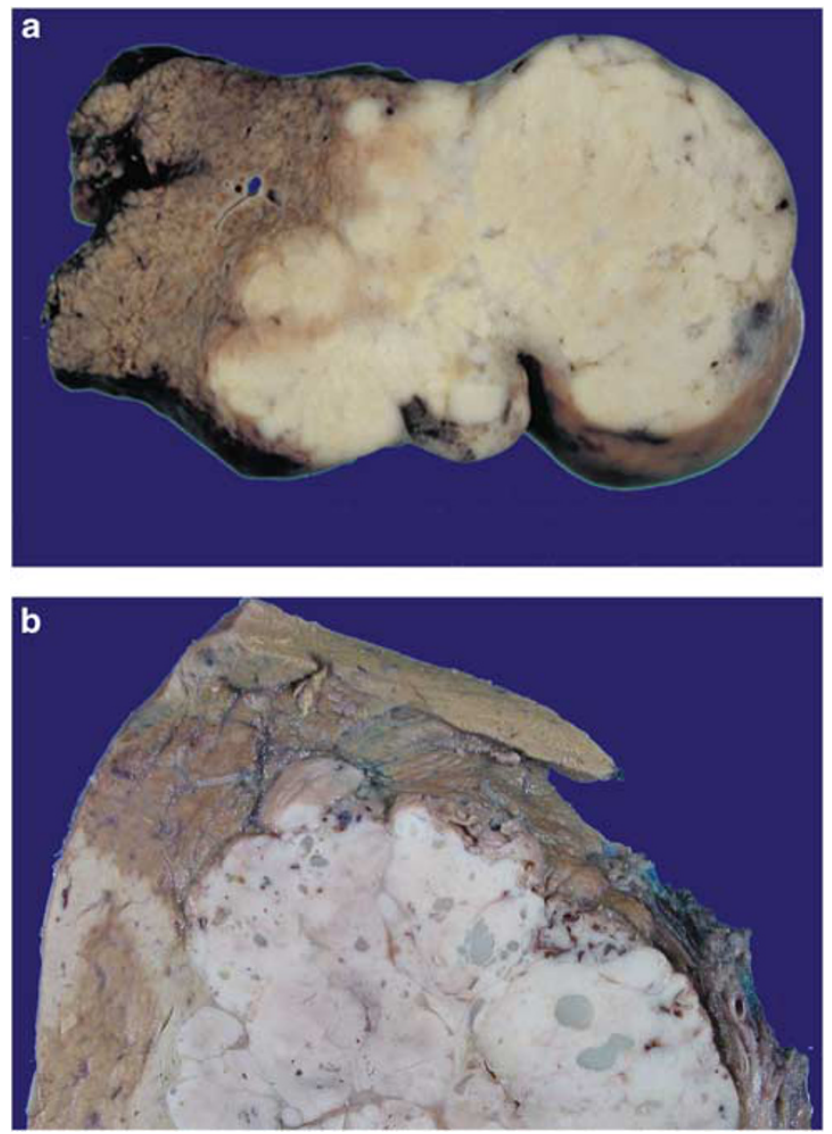

Figure 1 Gross features of acinar cell carcinomas of the liver. (a) Liver resection specimen showing a large well-demarcated tumor with a lobulated cut surface (case 1). (b) Cut surface of a resected liver tumor showing cystic spaces containing gelatinous secretions (case 4). magnification (Figure 2b). Occasional acini were cystically dilated and contained detached epithelial cells intermixed with a few histiocytes and eosinophilic secretion (Figure 2a). Bile secretion was not detected. A common feature was the gradual transition from well-formed acini to areas of less-organized acini that blended with solid areas (Figure 2c). The tumor cells were medium sized to large with basally located nuclei and distinct cell borders. The apical cytoplasm commonly showed faint PAS positivity, but intracellular mucin was not detected. Case 1 showed eosinophilic secretory bodies within the apical cytoplasm (Figure 2d). Two cases displayed predominantly a trabecular pattern (Figure 2e). Their nuclei were rounded and often contained a distinct nucleolus. Nuclear atypia was prominent in two tumors (Figure 2f). Mitoses were variable with counts ranging from $<4$ mitoses/10 high power fields (case 1) to significant counts of $\geq 10 / 10$ high power fields (other cases). Stromal hemosiderin was particularly evident in tumors with cystic dilated acini. Despite extensive sectioning, no additional components, in particular no areas of hepatocellular carcinoma, cholangiocarcinoma, hepatoblastoma or mesenchymal differentiation were seen. The surrounding liver parenchyma was unremarkable with no signs of chronic liver disease, steatosis or cirrhosis. No heterotopic pancreatic tissue or foci suggestive of pancreatic acinar metaplasia were seen.

\section{Immunohistochemical findings}

The tumor cells stained diffusely $(>70 \%)$ with pankeratin (KL-1) and CK 18, but more variably with epithelial membrane antigen (in 20-50\% tumor cells). All tumors expressed at least one of the three acinar cell markers trypsin, amylase and lipase. The expression of these enzymatic markers was typically focal and luminal (Figure 3a), but occasionally also strongly cytoplasmatic (Figure 3b). Two tumors stained focally with synaptophysin in $\leq 25 \%$ of tumor cells (Figure 3c) and chromogranin A. All tumors were negative for HepPar-1 (Figure 3d), CK7, CK19 (Figure 3d, subimage), CK20, CD56, AFP and CDX2. CD10 showed weak luminal staining in one case. No canalicular staining for CD10 or polyclonal CEA was detected in any of the cases. The proliferation fraction (MiB1) varied within the same tumor and from tumor to tumor with a range of $2-10 \%$.

\section{Discussion}

We observed four primary liver carcinomas whose features were identical with those of pancreatic acinar cell carcinoma. The tumors occurred in adults and affected both genders equally. The mean age of the patients (65 years) was slightly higher than that reported for pancreatic acinar cell carcinoma (58 years) and lower than the mean age for ductal adenocarcinoma (70 years). ${ }^{1,3}$ Three main questions 


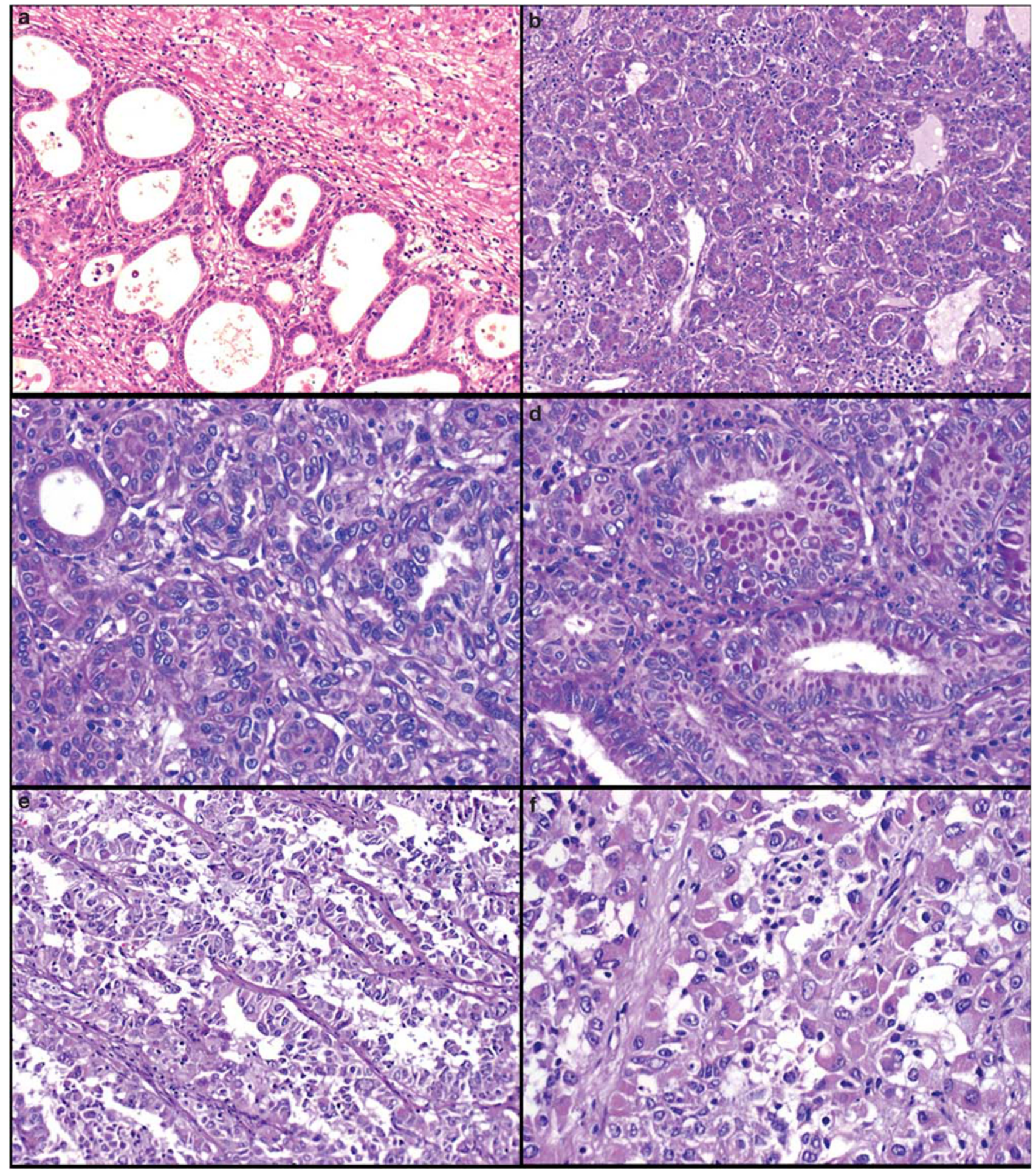

Figure 2 Histological features of acinar cell carcinoma of the liver. (a) Well-demarcated tumor lobule showing a microcystic acinar pattern. (b) Monotonous densely packed acinar structures. (c) Large acinar structures (upper left) merging with small acinar components. (d) Hyaline PAS-positive diastase-resistant secretory globules in the apical cytoplasm of tumor cells (case 1). (e) Trabecular and pseudoalveolar pattern (case 2). (f) Large tumor cells with eosinophilic cytoplasm and marked nuclear atypia (case 3).

have to be addressed in conjunction with the four neoplasms observed: first, do these tumors really represent true acinar cell carcinomas of pancreatic type, or are they merely unusual acinar variants of cholangiocarcinoma, hepatocellular carcinoma or adult hepatoblastoma? Second, can we safely exclude a solitary liver metastasis from an occult pancreatic acinar cell carcinoma, and third, how can 


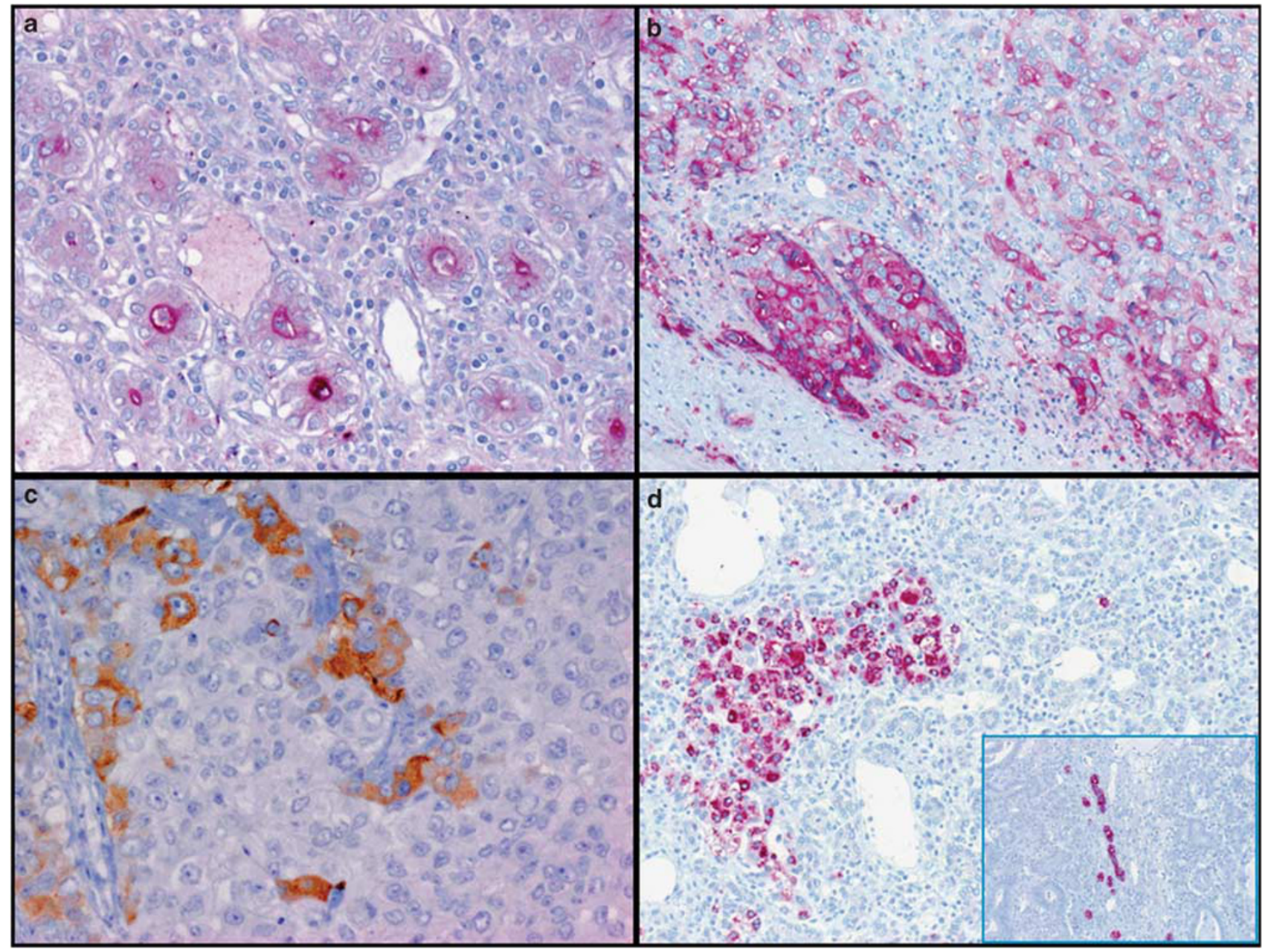

Figure 3 Immunohistochemical findings in acinar cell carcinoma of the liver. (a) Predominantly luminal expression of amylase (case 1). (b) Predominantly cytoplasmic expression of lipase (case 3). (c) Expression of synaptophysin in tumor cells (case 4). (d) Negativity for Hep-Par-1 (note strong staining in entrapped hepatocytes) and absence of the expression of cytokeratin 19 (inset; note expression of cytokeratin 19 in bile ductules next to unstained tumor cells).

we explain the histogenesis of these neoplasms, if they indeed represent primary hepatic acinar cell carcinomas?

All four liver tumors of our series showed features that clearly separated them from cholangiocarcinoma, hepatocellular carcinoma and hepatoblastoma. In contrast to cholangiocarcinoma and hepatocellular carcinoma, the tumors of our series arose in non-cirrhotic livers and none presented with significant jaundice or symptoms commonly associated with cholangiocarcinoma or hepatocellular carcinoma. Histologically, the presence of distinct acinar structures in a non-desmoplastic stroma, acini with apical cytoplasmic PAS-positivity, absence of bile plugs in irregular glands or pseudoglandular structures and generally less high-grade nuclear features are all criteria that argue against the diagnosis of one of the aforementioned tumors. Consistent with these histological features, the four hepatic acinar cell carcinomas lacked markers of biliary (CK7, CK19) and hepatocellular (Hep-Par-1, AFP, canalicular staining for CEA and CD10) differentiation. Instead they showed unequivocal staining (typically apical) with at least one of the acinar cell markers trypsin, amylase and lipase.

Adult hepatoblastoma may be another consideration when encountering a non-classifiable primary liver neoplasm in adults. ${ }^{13}$ However, the occurrence of hepatoblastoma in adults older than 30 years has been doubted by some authors. To date, $\sim 20$ cases of primary liver neoplasms diagnosed as hepatoblastomas in individuals older than 30 years have been published; most were mixed hepatoblastomas, with only four cases of purely epithelial hepatoblastoma. ${ }^{13,14}$ Although some areas in our cases revealed a monotonous pattern of immature-looking acini or gland-like structures that may superficially resemble fetal bile ducts suggesting a cholangioblastic hepatoblastoma, ${ }^{15}$ both the histological features and the immunohistochemical profiles exclude a biliary line of differentiation in our cases. 
Regarding the second issue, the exclusion of a metastasis from occult pancreatic acinar cell carcinoma, it is well known that a number of pancreatic acinar cell carcinomas initially present as metastasis, particularly in the liver, but also at unusual locations like the ovary, ${ }^{16}$ and are only subsequently identified in the pancreas. In our patients, however, there was no evidence of a pancreatic primary, neither on preoperative imaging procedures nor during intra-operative examination. In addition, none of the patients was found to have a pancreatic tumor on thorough postoperative diagnostic workup and none developed metastatic disease or showed evidence of a pancreatic tumor at a follow-up interval lasting up to 38 months. Another significant argument against a metastasis from a pancreatic primary is the fact that there was only one hepatic mass and not, as it is usually seen in metastatic pancreatic acinar cell carcinoma, multiple liver tumors. ${ }^{1-3,5}$ Moreover, metastatic pancreatic acinar cell carcinomas are usually large and about $50 \%$ of the patients with pancreatic acinar cell carcinoma have at the time of initial diagnosis evidence of metastatic disease, outside the liver. ${ }^{1-3,5}$

Regarding the histogenesis of hepatic acinar cell carcinoma, there is no ready answer as yet. Previous reports of extra-pancreatic acinar cell carcinomas suggested an origin from heterotopic or metaplastic pancreatic tissue, and this could be demonstrated in some cases. ${ }^{9-11}$ In the liver, ectopic pancreatic tissue is rare and neither in our cases nor in the previously reported one there was evidence of an origin from heterotopic pancreatic tissue. It is, however, known that both pancreatic and biliary tract epithelia develop from the same progenitor cells making an origin from non-committed biliary stem cells possible. ${ }^{17}$ Interestingly, a recent study by Kuo et $a l^{18}$ detected pancreatic acinar tissue in $4.2 \%$ of 382 liver explants. These pancreatic tissues showed expression of exocrine pancreatic markers including amylase, and were negative for biliary-type cytokeratins. Based on the close spatial relationship of the pancreatic tissue with reactive bile ductules and the apparent immunophenotypic transition from bile ductules to pancreatic acinar tissue, the authors favored a metaplastic origin from a hepatic progenitor cell lineage. ${ }^{18}$ On the other hand, acinar cell carcinoma of the pancreas occasionally produces AFP similar to hepatocellular carcinoma and a rare example of pancreatic neoplasm with hepatocellular differentiation has also been reported. ${ }^{19,20}$ These observations make it likely that a mechanism of 'transdifferentiation' might explain the origin of primary acinar cell carcinoma in the liver. Thus, hepatic acinar cell carcinomas may develop from non-committed progenitor cells that follow a pure pancreatic acinar cell differentiation. Preexisting heterotopic and/or metaplastic pancreatic acinar tissue may therefore not be necessary for the development of liver acinar cell carcinoma of the pancreatic type.
Pancreatic acinar cell carcinomas may include cells with neuroendocrine and ductal differentiation. $^{21}$ Cells expressing neuroendocrine markers such as synaptophysin and chromogranin A may be found scattered among the acinar tumor cells in up to $40 \%$ of the cases. ${ }^{5,22}$ Rarely, neuroendocrine cells are so numerous in acinar cell carcinomas that these neoplasms represent mixed acinar-endocrine carcinomas. ${ }^{21,22}$ Two of the hepatic acinar cell carcinomas of our series also contained neuroendocrine cells, indicating that the hepatic progenitor cells from which they probably originate have the same capacity to differentiate into neuroendocrine cell elements as their pancreatic counterparts.

Although it was initially thought that all pancreatic acinar cell carcinomas are aggressive neoplasms, it has become apparent that they occasionally may also follow an indolent clinical course with longterm survival. ${ }^{23,24}$ A large recent study showed an overall 5-year survival for pancreatic acinar cell carcinomas of $42.8 \%$ and a 5 -year survival of $22 \%$ for those with unresected acinar cell carcinomas. ${ }^{3}$ Our cases are also in line with a more favorable outcome, as none developed metastasis at a mean follow-up of 22 months and only one patient developed recurrent disease in the liver at 18 months.

In summary, we describe four patients with primary liver tumors that showed features identical with those of pancreatic acinar cell carcinomas. Recognition of our four cases during a period of only 4 years let us speculate that this unusual liver carcinoma might be more common than the scarcity of reports that have appeared so far suggests. Hepatic acinar cell carcinomas might have been significantly underrecognized owing to a limited familiarity of many surgical pathologists with pancreatic acinar cell carcinoma and not considering primary hepatic acinar cell carcinoma when evaluating liver neoplasms.

\section{Disclosure/conflict of interest}

The authors declare no conflict of interest.

\section{References}

1 Hruban RH, Pitman MB, Klimstra DS. AFIP Atlas of Tumor Pathology: Tumors of the Pancreas, 4th Series, Fascicle 6. American Registry of Pathology: Washington, D.C., 2007.

2 Kitagami H, Kondo S, Hirano S, et al. Acinar cell carcinoma of the pancreas: clinical analysis of 115 patients from Pancreatic Cancer Registry of Japan Pancreas Society. Pancreas 2007;35:42-46.

3 Wisnoski NC, Townsend Jr CM, Nealon WH, et al. 672 patients with acinar cell carcinoma of the pancreas: a population-based comparison to pancreatic adenocarcinoma. Surgery 2008;144:141-148.

4 Morohoshi T, Kanda M, Horie A, et al. Immunocytochemical markers of uncommon pancreatic tumors. Acinar cell carcinoma, pancreatoblastoma, and solid 
cystic (papillary-cystic) tumor. Cancer 1987;59: 739-747.

5 Klimstra DS, Heffess CS, Oertel JE, et al. Acinar cell carcinoma of the pancreas. A clinicopathologic study of 28 cases. Am J Surg Pathol 1992;16:815-837.

6 Sun Y, Wasserman PG. Acinar cell carcinoma arising in the stomach: a case report with literature review. Hum Pathol 2004;35:263-265.

7 Kawakami H, Kuwatani M, Onodera M, et al. Primary acinar cell carcinoma of the ampulla of Vater. J Gastroenterol 2007;42:694-697.

8 Chiaravalli AM, Finzi G, Bertolini V, et al. Colonic carcinoma with a pancreatic acinar cell differentiation. A case report. Virchows Arch 2009;455:527-531.

9 Makhlouf HR, Almeida JL, Sobin LH. Carcinoma in jejunal pancreatic heterotopia. Arch Pathol Lab Med 1999;123:707-711.

10 Mizuno Y, Sumi Y, Nachi S, et al. Acinar cell carcinoma arising from an ectopic pancreas. Surg Today 2007;37:704-707.

11 Ambrosini-Spaltro A, Potí O, De Palma M, et al. Pancreatic-type acinar cell carcinoma of the stomach beneath a focus of pancreatic metaplasia of the gastric mucosa. Hum Pathol 2009;40:746-749.

12 Hervieu V, Lombard-Bohas C, Dumortier J, et al. Primary acinar cell carcinoma of the liver. Virchows Arch 2008;452:337-341.

13 Altmann HW. Epithelial and mixed hepatoblastoma in the adult. Histological observations and general considerations. Pathol Res Pract 1992;188:16-26.

14 Kasper HU, Longerich T, Stippel DL, et al. Mixed hepatoblastoma in an adult. Arch Pathol Lab Med 2005;129:234-237.

15 Zimmermann A. Hepatoblastoma with cholangioblastic features ('cholangioblastic hepatoblastoma') and other liver tumors with bimodal differentiation in young patients. Med Pediatr Oncol 2002;39: 487-491.

16 Vakiani E, Young RH, Carcangiu ML, et al. Acinar cell carcinoma of the pancreas metastatic to the ovary: a report of 4 cases. Am J Surg Pathol 2008;32:1540-1545.

17 Deutsch G, Jung J, Zheng $\mathrm{M}$, et al. A bipotential precursor population for pancreas and liver within the embryonic endoderm. Development 2001;128: 871-881.

18 Kuo FY, Swanson PE, Yeh MM. Pancreatic acinar tissue in liver explants: a morphologic and immunohistochemical study. Am J Surg Pathol 2009;33:66-71.

19 Kawamoto S, Hiraoka T, Kanemitsu K, et al. Alphafetoprotein-producing pancreatic cancer-a case report and review of 28 cases. Hepatogastroenterology 1992; 39:282.

20 Cuilliere P, Lazure T, Bui M, et al. Solid adenoma with exclusive hepatocellular differentiation: a new variant among pancreatic benign neoplasms? Virchows Arch 2002;441:519-522.

21 Stelow EB, Shaco-Levy R, Bao F, et al. Pancreatic acinar cell carcinomas with prominent ductal differentiation: Mixed acinar ductal carcinoma and mixed acinar endocrine ductal carcinoma. Am J Surg Pathol 2010;34:510-518.

22 Ohike N, Kosmahl M, Klöppel G. Mixed acinarendocrine carcinoma of the pancreas. A clinicopathological study and comparison with acinar-cell carcinoma. Virchows Arch 2004;445:231-235.

23 Hashimoto M, Miki K, Kokudo N, et al. A long-term survivor of metastatic acinar cell carcinoma. Pancreas 2007;34:271-272.

24 Antoine M, Khitrik-Palchuk M, Saif MW. Long-term survival in a patient with acinar cell carcinoma of pancreas. A case report and review of literature. JOP 2007;8:783-789. 